\title{
Immunohistochemical detection of ZAP70 in chronic lymphocytic leukemia predicts immunoglobulin heavy chain gene mutation status and time to progression
}

Joan H Admirand ${ }^{1}$, Ronald J Knoblock ${ }^{1}$, Kevin R Coombes ${ }^{2}$, Constantine Tam ${ }^{3}$, Ellen J Schlette ${ }^{1}$, William G Wierda ${ }^{3}$, Alessandra Ferrajoli ${ }^{3}$, Susan O’Brien ${ }^{3}$, Michael J Keating ${ }^{3}$, Rajyalakshmi Luthra ${ }^{1}$, L Jeffrey Medeiros ${ }^{1}$ and Lynne V Abruzzo ${ }^{1}$

${ }^{1}$ Department of Hematopathology, University of Texas M.D. Anderson Cancer Center, Houston, TX, USA; ${ }^{2}$ Department of Bioinformatics and Computational Biology, University of Texas M.D. Anderson Cancer Center, Houston, TX, USA and ${ }^{3}$ Department of Leukemia, University of Texas M.D. Anderson Cancer Center, Houston, TX, USA

\begin{abstract}
Zeta-associated protein-70 (ZAP70) expression measured by flow cytometry has been proposed as a surrogate marker of the somatic mutation status of the immunoglobulin heavy chain variable region (IGHV) genes in chronic lymphocytic leukemia. However, attempts to implement this approach in clinical flow cytometry laboratories have been problematic; many commercially available antibodies give unreliable results. Assessment of ZAP70 protein expression by immunohistochemistry in chronic lymphocytic leukemia tissue sections is an easy, alternative approach, although lack of quantitation and subjective interpretation of results are potential pitfalls. In this study, we correlated ZAP70 protein expression, assessed by immunohistochemistry, with ZAP70 messenger RNA (mRNA) transcript expression, assessed by semi-quantitative real-time reverse transcriptase-polymerase chain reaction assay, with the somatic mutation status of the IGHV genes in previously untreated patients with chronic lymphocytic leukemia. Expression of ZAP70 protein and mRNA transcripts correlated strongly $\left(P=8.238 \times 1^{-12}\right)$. Expression of ZAP70 protein and mRNA transcripts also correlated strongly with the somatic mutation status of the IGHV genes $(P=0.000071$ and $P=0.00076$, respectively). Further, ZAP70 positivity by immunohistochemistry was associated with an increased risk of progression to therapy requirement (3-year risk $83 \%$ vs $31 \%$ for ZAP70 negative by immunohistochemistry, $P=0.03$ ). These results show that $Z A P 70$ expression assessed by immunohistochemistry is a reliable surrogate marker of the somatic mutation status of the IGHV genes, and predicts time to progression.

Modern Pathology (2010) 23, 1518-1523; doi:10.1038/modpathol.2010.131; published online 23 July 2010
\end{abstract}

Keywords: chronic lymphocytic leukemia; immunoglobulin heavy chain variable region genes; immunohistochemistry; QRT-PCR; somatic mutation status; ZAP70

Zeta-associated protein-70 (ZAP70), a member of the Syk family of tyrosine kinases, has an important role in T-cell receptor signaling, natural killer cell activation, and early B-cell development. ${ }^{1}$ ZAP70

Correspondence: Dr LV Abruzzo, MD, PhD, Department of Hematopathology, University of Texas M.D. Anderson Cancer Center, Box 350, 1515 Holcombe Boulevard, Houston, TX 77030, USA.

E-mail: labruzzo@mdanderson.org

Received 21 February 2010; revised 23 June 2010; accepted 28 June 2010; published online 23 July 2010 protein is not expressed in most normal mature B cells, but is expressed in various B- and T-cell lymphomas. ${ }^{2-4}$ In previous studies, ZAP70 protein expression measured by flow cytometry was associated with unmutated immunoglobulin heavy chain variable region (IGHV) genes and poorer clinical outcome in patients with chronic lymphocytic leukemia. ${ }^{5-11}$ Subsequent attempts to implement ZAP70 analysis in clinical flow cytometry laboratories, however, have been have been fraught with difficulty; this approach is technically challenging and results often have been unreliable. 
Detection of ZAP70 protein by immunohistochemistry using tissue sections of chronic lymphocytic leukemia is an easy and inexpensive technique. Potential criticisms of this approach, however, are that immunohistochemistry is not quantitative and that interpretation is somewhat subjective. To address these issues, we compared immunohistochemistry for $Z A P 70$ protein with the presence of $Z A P 70$ messenger RNA (mRNA) transcripts measured by a semi-quantitative reverse transcriptase-polymerase chain reaction (QRTPCR) assay. We show that detection of ZAP70 protein assessed by immunohistochemistry strongly correlates with ZAP70 mRNA expression assessed by QRT-PCR assay, and that the results of both methods correlate with the somatic mutation status of the IGHV genes in chronic lymphocytic leukemia. Further, our results show that ZAP70 protein assessed by immunohistochemistry predicts time to progression.

\section{Materials and methods}

\section{Patient Characteristics}

The Institutional Review Board at The University of Texas M.D. Anderson Cancer Center approved this study. Informed consent was provided according to the Declaration of Helsinki. Sixty-three patients with chronic lymphocytic leukemia, according to the criteria of the World Health Organization Classification system, ${ }^{12}$ were included in this study. The clinical characteristics of these patients are summarized in Table 1 . We selected patients based on the availability of clinical material and the completeness of long-term follow-up. All patients were previously untreated at the time of IGHV sequence analysis and ZAP70 testing. The median age of patients was 58 years (range 27-76 years) and

Table 1 Clinical characteristics

\begin{tabular}{lc}
\hline Characteristic & Median (range) \\
\hline Age (years) & $58(27-76)$ \\
Male gender & $44(70 \%)$ \\
Date of test & August 2000 to \\
& November 2004 \\
Diagnosis to test (months) & $26(1-129)$ \\
& \\
Rai stage 0 & $6(10 \%)$ \\
Rai stage 1-2 & $50(79 \%)$ \\
Rai stage 3-4 & $7(11 \%)$ \\
& \\
Hemoglobin (g/dl) & $13.0(9.9-17.0)$ \\
White cell count ( $\times 109 / 1)$ & $35.7(7.5-255.0)$ \\
Platelets ( $\times 109 / 1)$ & $177(39-351)$ \\
B2-microglobulin (mg/l) & $3.0(1.3-7.4)$ \\
& \\
Unmutated IGHV & $33 / 63(52 \%)$ \\
ZAP70 immunohistochemistry positive & $36 / 63(57 \%)$ \\
ZAP70 QRT-PCR assay positive & $39 / 60(65 \%)$ \\
ZAP70 flow cytometry positive & $24 / 48(50 \%)$ \\
\hline
\end{tabular}

$70 \%$ were males (44 men and 19 women). The majority were in modified intermediate-risk stage (Rai 1/2, 79\%), with low- and high-risk stages constituting 10 and $11 \%$ of patients, respectively. For the analysis of time to progression, we defined the start date as the date the bone marrow sample, which was tested for ZAP70 expression, was obtained from the patient for evaluation of chronic lymphocytic leukemia at our institution. At the time of testing, patients were a median of 26 months (range 1-129 months) from initial diagnosis by history.

Forty-one patients (65\%) had symptomatic and/or advanced disease meeting National Cancer Institute (NCI) sponsored guidelines for treatment, ${ }^{13}$ and received therapy within 6 weeks of IGHV sequence analysis and ZAP70 testing. The remaining 22 patients were evaluable for disease progression: four patients received rituximab as a phase II study of early intervention ${ }^{14}$ without meeting NCI criteria for therapy, and were censored at the time of rituximab administration; seven received fludarabine, cyclophosphamide and rituximab (FCR) with $(n=1)$ or without $(n=6)$ alemtuzumab; five received rituximab with $(n=1)$ or without $(n=4)$ alemtuzumab; and six remained without requirement for therapy at 21-77 months of follow-up. Ten patients died during follow-up, and median survivor follow-up was 42 months (range 29-84 months).

\section{Assessment of ZAP70 Protein Expression by Immunohistochemistry and Flow Cytometry}

Immunohistochemical staining for ZAP70 protein was performed in all 63 patients using routinely fixed and processed paraffin-embedded tissue sections of bone marrow core biopsies and/or clot sections and a specific monoclonal antibody (Upstate Cell Signaling Systems, Lake Placid, NY, USA), as described previously. ${ }^{2}$ All cases were scored independently by two hematopathologists (RK and JA) without knowledge of the IGHV somatic mutation status. Cases were scored as 0 (negative), $1+$ (weakly positive; granular cytoplasmic staining with nuclear blush in the majority of tumor cells), or $2+$ (strongly positive in the majority of tumor cells, approximating the expression of normal $\mathrm{T}$ cells) (Figure 1). For statistical analysis, cases with $1+$ and $2+$ expression were combined and designated as positive. For all cases, there was agreement with respect to positivity or negativity. The flow cytometry assay for $Z A P 70$ protein expression was performed by the Chronic Lymphocytic Leukemia Research Consortium laboratory, as described previously. ${ }^{15}$

\section{Isolation of Total RNA and Evaluation of IGHV Genes}

Total RNA was extracted from purified chronic lymphocytic leukemia cells isolated from peripheral 


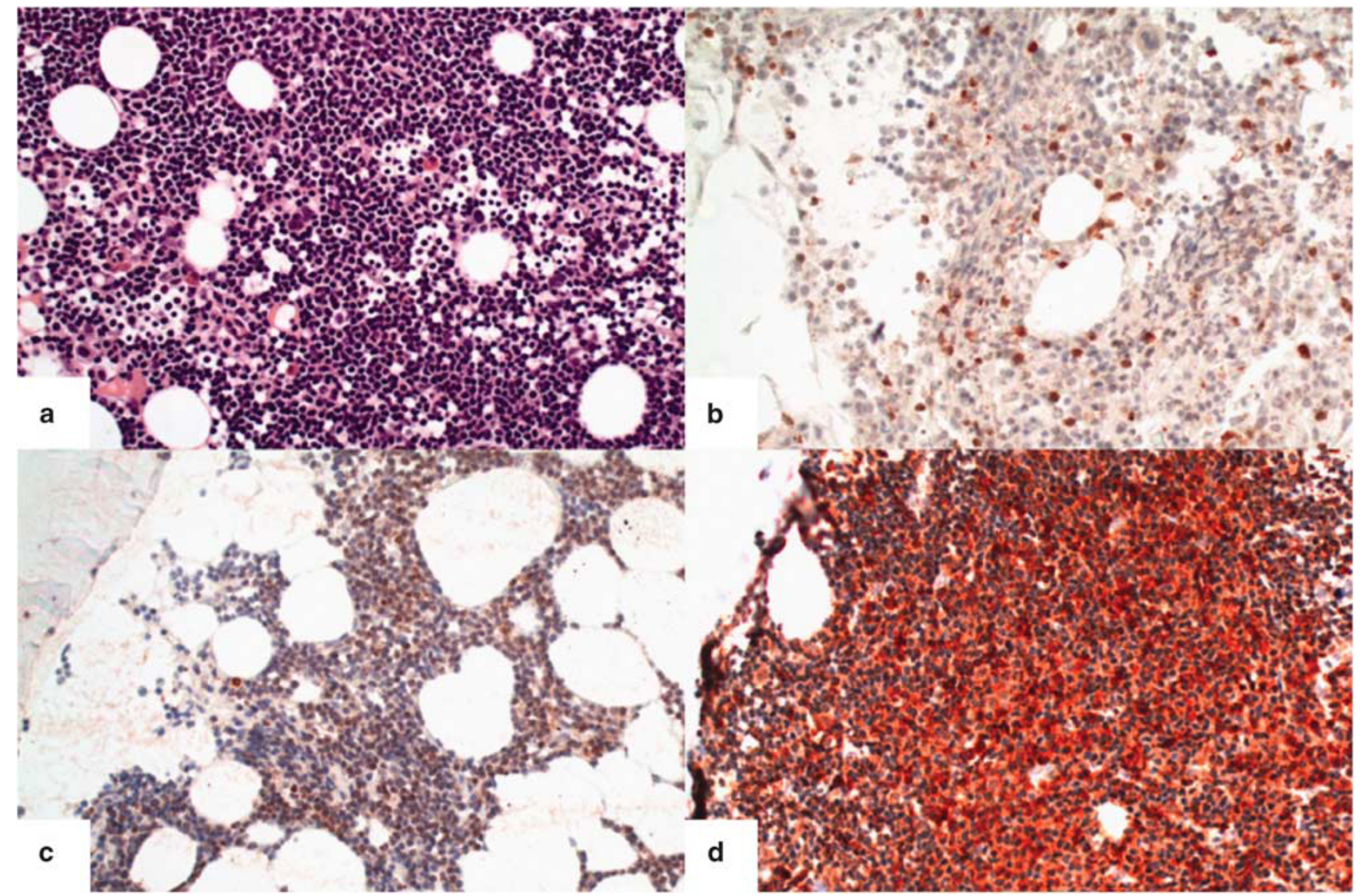

Figure 1 Immunohistochemistry for ZAP70 in chronic lymphocytic leukemia involving bone marrow. (a) A representative case showing an interstitial infiltrate of small, mature-appearing lymphoid cells (hematoxylin and eosin, $\times 200$ ). (b) In this case, the chronic lymphocytic leukemia cells are negative for ZAP70, but interspersed benign T cells are strongly positive. (c) In this case, the chronic lymphocytic leukemia cells show weak $(1+) Z A P 70$ staining. (d) In this case, the chronic lymphocytic leukemia cells show strong $(2+)$ ZAP70 staining. (b-d, immunohistochemistry with hematoxylin counterstain, $\times 200$ ).

blood. $^{6}$ All preparations contained $\geq 97 \%$ cells positive for CD5 and CD19. The IGHV somatic mutation status was determined as described previously. ${ }^{6}$ Cases were designated as unmutated if there were fewer than $2 \%$ mutations ( $\geq 98 \%$ homology to germ-line sequences), or as mutated if there were $\geq 2 \%$ mutations $(<98 \%$ homology to germ-line sequences) compared with the germ-line sequences. ${ }^{16}$

\section{Semi-Quantitative RT-PCR Assay}

ZAP70 mRNA transcripts were assessed by a QRTPCR assay using TaqMan $\AA$ technology and a PRISM 7000 Sequence Detector (Applied Biosystems, Foster City, CA, USA) in 60 patients for whom sufficient total RNA was available. Each PCR reaction $(25 \mu \mathrm{l})$ contained $50 \mathrm{ng}$ cDNA, $1 \times$ TaqMan (R Universal PCR Master Mix without AmpErase $\AA$ UNG, unlabeled PCR primers, specific ZAP70 primers, and a 6-carboxy fluorescein $\left(\mathrm{FAM}^{\mathrm{TM}}\right)$-labeled TaqMan $\AA$ MGB probe. The $Z A P 70$ primers were designed from sequences in exons 2 and 3 (Assays-on-Demand Gene Expression system,
Applied Biosystems). Amplification of $18 \mathrm{~S}$ ribosomal RNA (rRNA) was performed in all cases to normalize $Z A P 70$ values. The $18 \mathrm{~S}$ rRNA probe is

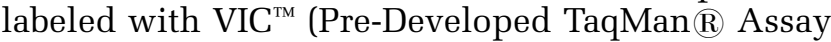
Reagents, Applied Biosystems). After incubation at $95^{\circ} \mathrm{C}(10 \mathrm{~min})$, the cDNA was amplified for 40 cycles of denaturation $\left(95^{\circ} \mathrm{C}\right.$ for $\left.15 \mathrm{~s}\right)$ and combined annealing/extension $\left(60^{\circ} \mathrm{C}\right.$ for $\left.1 \mathrm{~min}\right)$. Each sample was analyzed in duplicate. Expression was categorized as positive ( $\leq 25$ cycles) or negative $(>25$ cycles). We determined the cycle number cut points based on the Martingale residuals from a null Cox proportional hazards model predicting time to treatment in a series of 92 patients; 25 cycles marks the cut point for $Z A P 70$ mRNA expression.

\section{Results}

Immunohistochemical staining for $Z A P 70$ protein in a total of 63 cases demonstrated that 27 cases were negative for $Z A P 70$ expression and 36 were positive (Figure 1). There was a strong correlation between ZAP70 expression assessed by immunohistochemistry and QRT-PCR assay in the 60 chronic 
Table 2a $Z A P 70$ expression assessed by immunohistochemistry and QRT-PCR assay

\begin{tabular}{lcc}
\hline Immunohistochemistry & QRT-PCR- & QRT-PCR+ \\
\hline Negative & $21 / 26(81 \%)$ & $5 / 26(19 \%)$ \\
Positive & $0 / 34(0 \%)$ & $34 / 34(100 \%)$
\end{tabular}

$P=8.238 \times 10^{-12}$.

Table 2b IGHV mutation status and ZAP70 expression assessed by QRT-PCR assay

\begin{tabular}{lrr}
\hline IGHV & QRT-PCR- & QRT-PCR+ \\
\hline Mutated & $15 / 26(62 \%)$ & $11 / 26(38 \%)$ \\
Unmutated & $5 / 34(15 \%)$ & $29 / 34(85 \%)$
\end{tabular}

$P=0.00076$.

Table 2c IGHV mutation status and ZAP70 expression assessed by immunohistochemistry

\begin{tabular}{lcc}
\hline IGHV & Immunohistochemistry- & Immunohistochemistry+ \\
\hline Mutated & $19 / 28(71 \%)$ & $9 / 28(29 \%)$ \\
Unmutated & $6 / 35(20 \%)$ & $29 / 35(80 \%)$ \\
\hline
\end{tabular}

$P=0.000071$.

lymphocytic leukemia cases for which sufficient total RNA was available to perform the QRT-PCR assay $\left(P=8.2 \times 10^{-12}\right.$ Fisher's exact test) (Table 2a). All cases that were positive by immunohistochemistry were also positive by QRT-PCR assay (34 of 34 cases); 21 of 26 (81\%) cases negative by immunohistochemistry were also negative by QRT-PCR assay. The discordant cases (5 of 26, 19\%) were positive by QRT-PCR assay, but negative by immunohistochemistry.

Of the 63 cases, 30 cases had mutated and 33 had unmutated IGHV genes. The somatic mutation status correlated significantly with ZAP70 expression assessed by QRT-PCR assay and immunohistochemistry $\quad(P=0.00076 \quad$ and $P=0.000071$, respectively, Fisher's exact test) (Tables $2 \mathrm{~b}$ and $2 \mathrm{c}$ ). Most unmutated cases were positive for $Z A P 70$ by QRT-PCR assay (29 of 34 cases, 85\%) (Table 2b) and immunohistochemistry (29 of 35 cases, $83 \%$ ) (Table 2c). Most mutated cases were negative for ZAP70 by QRT-PCR assay (15 of 26 cases, 58\%) (Table 2b) and immunohistochemistry (19 of 28 cases, 68\%) (Table 2c). Of the nine mutated cases that expressed ZAP7O by immunohistochemistry, two belonged to the VH family 3-21.

Nineteen cases showed discordance between IGHV somatic mutation status and ZAP70 expression assessed by immunohistochemistry and/or QRT-PCR assay. Of the 13 discordant cases with mutated IGHV genes, eight were positive for $Z A P 70$ expression by both immunohistochemistry and QRT-PCR assay, and one was positive by immuno- histochemistry, but unevaluable by QRT-PCR assay. The remaining four cases were positive by QRTPCR assay, but negative by immunohistochemistry. Of the six discordant cases with unmutated IGHV genes, five were negative for $Z A P 70$ expression by both immunohistochemistry and QRT-PCR assay. The remaining case was positive by QRT-PCR assay, but negative by immunohistochemistry.

\section{Correlation with Clinical Outcome}

Twenty-two patients not requiring immediate therapy ( $\leq 6$ weeks) were evaluable for disease progression. ZAP70 positivity by immunohistochemistry was associated with increased risk of progression to therapy requirement (3-year risk $83 \%$ vs $31 \%$ for $Z A P 70$ negative by immunohistochemistry, $P=0.03$; Figure 2a). $Z A P 70$ positivity by QRT-PCR assay and flow cytometry showed a similar trend, with lesser degrees of statistical significance $(P=0.15$ and 0.08 for QRT-PCR assay and flow cytometry, respectively) (data not shown). In contrast, the association between unmutated IGHV status and progression to therapy was weak $(P=0.27$; Figure $2 b)$. Notably, two of the four patients with mutated IGHV patients who progressed to therapy requirement showed discordant ZAP70 positivity by immunohistochemistry.

The rate of complete remission for the 41 patients who received FCR therapy was not significantly different between ZAP70-positive and -negative patients $(P=0.45,0.70$, and 1.0 for immunohistochemistry, QRT-PCR assay, and flow cytometry, respectively), nor was it different between patients with unmutated and mutated IGHV genes $(P=0.28)$. Neither ZAP70 expression nor IGHV mutation status affected survival within the current duration of follow-up ( $P>0.35$ for all comparisons).

\section{Discussion}

The initial studies of the association between $Z A P 70$ protein expression by flow cytometry and IGHV somatic mutation status demonstrated a high degree of concordance. In the study by Crespo et al, ${ }^{9}$ all 21 mutated cases were negative for ZAP70, and the majority of unmutated cases (32 of $35,91 \%$ ) were positive for $Z A P 70$. A few unmutated cases ( 3 of 35 , $9 \%$ ) were negative for $Z A P 70$ by flow cytometry, but weakly expressed ZAP70 protein evaluated by western blot assay. In a larger study of 167 patients by Orchard et al, ${ }^{8}$ a small subset of mutated cases expressed $Z A P 70$ protein (5\%), and a small subset of unmutated cases $(13 \%)$ were negative. Since then, other studies have demonstrated a higher degree of discordance between $Z A P 70$ protein expression and IGHV somatic mutation status. In these studies, the percentage of unmutated cases that fail to express $Z A P 70$, by either flow cytometry or immunohistochemistry, has ranged from 6 to $29 \%$, and the percentage of mutated cases that express ZAP7O 

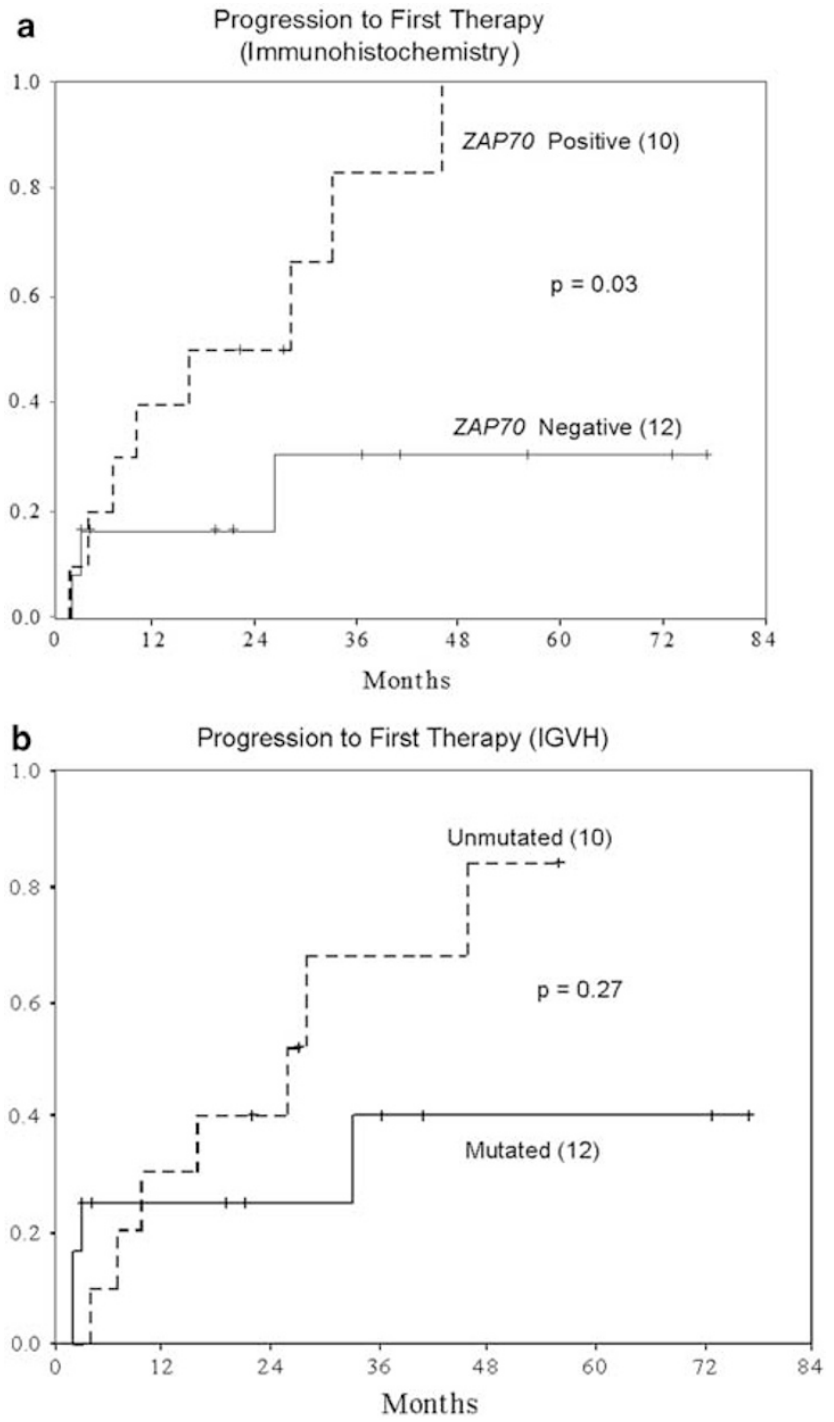

Figure 2 Progression to first therapy. (a) ZAP70 positivity by immunohistochemistry was associated with increased risk of progression to therapy requirement $(n=22$; 3-year risk $83 \%$ vs $31 \%$ for $Z A P 70$ negative by immunohistochemistry, $P=0.03$ ). (b). In contrast to $Z A P 70$ positivity by immunohistochemistry, the association between unmutated IGHV status and progression to therapy was weak $(n=22 ; P=0.27)$.

has ranged from 5 to $32 \% .^{2,3,9,10,15,17-19}$ The degree of discordance between ZAP70 expression and IGHV mutation status observed in our study is consistent with these findings. By immunohistochemistry, we found that $20 \%$ of unmutated cases failed to express $Z A P 70$, and $29 \%$ of mutated cases expressed $Z A P 70$. Two of the mutated cases that expressed $Z A P 70$ by immunohistochemistry belonged to the VH3-21 family, which has been associated with a poor prognosis and expression of $Z A P 70 .{ }^{18}$ By QRTPCR assay, we found that $15 \%$ of unmutated cases failed to express $Z A P 70 \mathrm{mRNA}$ transcripts, and $38 \%$ of mutated cases expressed ZAP70 mRNA transcripts. We found that no cases that were positive for
ZAP70 by immunohistochemistry, but negative by QRT-PCR assay. However, there were a small subset of cases that were negative by immunohistochemistry, but positive by QRT-PCR assay, presumably because of the greater sensitivity of the QRT-PCR assay compared with immunohistochemistry.

As the stability of ZAP7O expression and IGHV mutation status in cases that have undergone clonal evolution over the disease course and therapeutic interventions is unclear, we chose to focus our study on previously untreated patients. Consistent with data presented by other investigators using flow cytometry-based assays of $Z A P 70$ protein expression, ${ }^{9,15}$ our study shows that patients with $Z A P 70$ positive chronic lymphocytic leukemia have more aggressive disease with earlier time to therapy requirement. Although immunohistochemistry was the only method associated with a significant $P$-value, all three methods showed the same trend. The results of the QRT-PCR and flow cytometry assays may have been affected by the smaller number of samples available for analysis $(n=60$ and 48 , respectively).

Initial research studies demonstrated that ZAP70 protein expression by flow cytometry is a reliable surrogate marker of IGHV gene mutation status. However, introduction of this assay into the diagnostic flow cytometry laboratory as a clinical test has been problematic. ${ }^{19}$ ZAP70 protein is a labile, intracellular antigen that is weakly expressed by chronic lymphocytic leukemia cells. Furthermore, assessment of ZAP70 protein using commercially available antibodies obtained from different vendors has correlated poorly with IGHV gene mutation status. To address this problem, Bakke et $a 1^{20}$ proposed a ratio metric method to normalize this effect. Others have recommended using a secondary antibody. ${ }^{8}$ An inherent advantage of immunohistochemistry is its applicability to paraffin-embedded specimens, eliminating the need for stored fresh specimens. It is particularly useful in retrospective analyses of clinical trials that were completed before assays for ZAP70 became available. Within our relatively small patient cohort, the performance of ZAP70 immunohistochemistry was at least equal to that of QRT-PCR and flow cytometry assays in its correlation with IGHV mutation status and its association with disease progression.

\section{Acknowledgement}

This work was supported in part by grants from the CLL Global Research Foundation (LVA) and the National Cancer Institute (LVA, 5R01CA123252-3).

\section{Disclosure/conflict of interest}

The authors declare no conflict of interest. 


\section{References}

1 Schweighoffer E, Vanes L, Mathiot A, et al. Unexpected requirement for ZAP-70 in pre-B cell development and allelic exclusion. Immunity 2003;18: 523-533.

2 Admirand JH, Rassidakis GZ, Abruzzo LV, et al. Immunohistochemical detection of ZAP-70 in 341 cases of non-Hodgkin and Hodgkin lymphoma. Mod Pathol 2004;17:954-961.

3 Carreras J, Villamor N, Colomo L, et al. Immunohistochemical analysis of ZAP-70 expression in B-cell lymphoid neoplasms. J Pathol 2005;205:507-513.

4 Sup SJ, Domiati-Saad R, Kelley TW, et al. ZAP-70 expression in B-cell hematologic malignancy is not limited to CLL/SLL. Am J Clin Pathol 2004;122: 582-587.

5 Kipps TJ. Chronic lymphocytic leukemia. Curr Opin Hematol 2000;7:223-234.

6 McCarthy $\mathrm{H}$, Wierda WG, Barron LL, et al. High expression of activation-induced cytidine deaminase (AID) and splice variants is a distinctive feature of poor-prognosis chronic lymphocytic leukemia. Blood 2003;101:4903-4908.

7 Hamblin TJ, Davis Z, Gardiner A, et al. Unmutated Ig $\mathrm{V}(\mathrm{H})$ genes are associated with a more aggressive form of chronic lymphocytic leukemia. Blood 1999;94: 1848-1854.

8 Orchard JA, Ibbotson RE, Davis Z, et al. ZAP-70 expression and prognosis in chronic lymphocytic leukaemia. Lancet 2004;363:105-111.

9 Crespo $\mathrm{M}$, Bosch $\mathrm{F}$, Villamor $\mathrm{N}$, et al. ZAP-70 expression as a surrogate for immunoglobulinvariable-region mutations in chronic lymphocytic leukemia. N Engl J Med 2003;348:1764-1775.

10 Wiestner A, Rosenwald A, Barry TS, et al. ZAP-70 expression identifies a chronic lymphocytic leukemia subtype with unmutated immunoglobulin genes, inferior clinical outcome, and distinct gene expression profile. Blood 2003;101:4944-4951.
11 Chen L, Widhopf G, Huynh L, et al. Expression of ZAP70 is associated with increased B-cell receptor signaling in chronic lymphocytic leukemia. Blood 2002;100:4609-4614.

12 Muller-Hermelink HKCD, Montserrat E, Harris NL. Chronic lymphocytic leukemia/small lymphocytic lymphoma In: Jaffe ES HN, Stein H, Vardiman JW (eds). Pathology and Genetics of Tumours of Haematopoietic and Lymphoid Tissue Vol. IARC Press: Lyon, 2001, pp, 127-130.

13 Cheson BD, Bennett JM, Grever M, et al. National Cancer Institute-sponsored Working Group guidelines for chronic lymphocytic leukemia: revised guidelines for diagnosis and treatment. Blood 1996;87:4990-4997.

14 Thomas DA, O’Brien S, Giles FJ, et al. Single-agent rituximab in early-stage chronic lymphocytic leukemia. Oncology 2002;16:164a.

15 Rassenti LZ, Huynh L, Toy TL, et al. ZAP-70 compared with immunoglobulin heavy-chain gene mutation status as a predictor of disease progression in chronic lymphocytic leukemia. N Engl J Med 2004;351:893-901.

16 Fais F, Ghiotto F, Hashimoto S, et al. Chronic lymphocytic leukemia B cells express restricted sets of mutated and unmutated antigen receptors. J Clin Invest 1998;102:1515-1525.

17 Oppezzo P, Vasconcelos Y, Settegrana C, et al. The LPL/ADAM29 expression ratio is a novel prognosis indicator in chronic lymphocytic leukemia. Blood 2005;106:650-657.

18 Krober A, Bloehdorn J, Hafner S, et al. Additional genetic high-risk features such as $11 \mathrm{q}$ deletion, 17p deletion, and V3-21 usage characterize discordance of ZAP-70 and VH mutation status in chronic lymphocytic leukemia. J Clin Oncol 2006;24:969-975.

19 Orchard J, Ibbotson R, Best G, et al. ZAP-70 in B cell malignancies. Leuk Lymphoma 2005;46:1689-1698.

20 Bakke AC, Purtzer Z, Leis J, et al. A robust ratio metric method for analysis of Zap-70 expression in chronic lymphocytic leukemia (CLL). Cytometry B Clin Cytom 2006;70:227-234. 